\title{
FREQUENCY OF GLUCOSE-6-PHOSPHATE DEHYDROGENASE (G6PD) DEFICIENCY AMONG FEBRILE PATIENTS IN MALARIA-ENDEMIC COMMUNITIES IN SOUTHWESTERN NIGERIA
}

\author{
${ }^{1}$ Nassar, S. A., ${ }^{2}$ Olayiwola, J. O. and ${ }^{1}$ Ogunmola, O. S. \\ ${ }^{1}$ Department of Medical Laboratory Sciences, Ladoke Akintola University of Technology, Ogbomoso, Nigeria \\ ${ }^{2}$ Department of Biological Sciences, Ajayi Crowther University, Oyo State, Nigeria. \\ Corresponding author's email address: jo.olayiwola@acu.edu.ng,olusolajohn97@yahoo.com \\ (Received: $28^{\text {th }}$ June, 2018; Accepted: $16^{\text {th }}$ November, 2018)
}

\section{ABSTRACT}

\begin{abstract}
Glucose-6-phosphate dehydrogenase (G6PD) enzyme plays an active role in survival of erythrocytes. The deficiency of G6PD in the red blood cells is a clinical problem attributed to neonatal jaundice and chronic hemolytic anemia. Three hundred and thirty three blood samples were collected from consenting participants and screened for G6PD using methaemoglobin reductase method and malaria parasite microscopically by using Giemsa staining technique. The data obtained were analyzed with SPSS (version 16) software as statistical tool with $p$ value $\leqslant 0.05$ as level of significance. The prevalence of G6PD deficiency in male and female participants was $27.2 \%$ and $19.2 \%$ respectively. There was no significant association between G6PD deficiency and sex (0.086). Fifty 50 (15\%) of samples were positive for malaria (P. falciparum associated) with prevalence rate of $10.2 \%$ among female as against $4.6 \%$ found among male counterparts. There was significant association between malaria and gender $(\mathrm{p}$-value $=0.04)$. Also, the rate of G6PD was higher in male $(27.2 \%)$ than in female $(19.5 \%)$ participants but not statistically significant (0.086). Low level of both G6PD deficiency and malaria (2.4\%) was found. Due to possibility of G6PD deficiency, there is need for the diagnosis of G6PD in the management of malaria.
\end{abstract}

\section{INTRODUCTION}

Glucose-6-phosphate dehydrogenase (G6PD) deficiency is one of the known inherited human enzyme diseases. It affects $10 \%$ of the world population, which amounts to $200-400$ million people globally (Cappellini and Fiorelli 2008; Williams et al., 2013). G6PD deficiency results in 3,400 and 4,100 deaths in 1990 and 2013 respectively (GBD, 2013). It is an X-linked disorder genetically transmitted by a sex-linked gene of intermediate dominance causing hemolytic anemia and neonatal jaundice (Beutler, 1983; Viroj, 2005).

Deficiency of G6PD enzyme in the red blood cells, under certain circumstances, may lead to an abnormal rupture of the membrane of red blood cell with resultant hemolytic anemia (Lukens and Glader, 1999). The enzyme for G6PD is known to play an active role in the survival of erythrocytes. In pentose phosphate pathway of erythrocyte, G6PD is an enzyme that is involved in the formation of NADPH, which is useful in the generation of reduced glutathione (GSH). GSH produced can then be used by red blood cells. Oxidative stress can be induced in erythrocyte whose G6PD enzymes are deficient. In this case, GSH is not produced and $\mathrm{H}_{2} \mathrm{O}_{2}$ is not reduced to $\mathrm{H}_{2} \mathrm{O}$, leading to oxidative stress and hemolysis (Williams et al., 2013).

The G6PD deficiency of red blood cells is a health problem in developing countries, responsible for neonatal jaundice and, depending on the individual magnitude of deficiency, chronic hemolytic anemia and hemolytic attacks after the ingestion of certain oxidants (Luzzatto, 2010; Sutherland et al., 2010). In epidemiological studies, it has been shown that the prevalence of G6PD deficiency is significantly related to malaria. Malaria is known as a parasitic disease that affects $300-500$ million people all over the world. It is widespread in tropical and subtropical regions of Asia, Africa and American continents (Williams et al., 2013).

Five different types of Plasmodium species (P. falciparum, P. vivax, P. ovalae, P. malariae and P. knowlesi) are responsible for malaria by infecting erythrocytes (Bello et al., 2016). It can become a lifethreatening condition when it is not treated or when it occurred in anaemic patients. Each year, 
malaria leads to deaths of millions of people all around the world and a large percentage of deaths are reported in Sub-Saharan regions of Africa. It has been reported that malaria and G6PD deficiency share the same geographic distribution (Haworth et al., 1988). It was shown that G6PD enzyme has various genetic variants and polymorphic frequencies. Highly polymorphic frequencies, which are indicators of G6PD deficiency, are noticed in malaria-endemic regions such as Asia, Africa, Central and South America, while the rate decreases in non-endemic regions. This suggests the relationship between G6PD deficiency and malaria (Haworth et al., 1988; WHO, 2009). Therefore, the aim of this study is to investigate the relationship between G6PD deficiency and malaria among patients attending teaching hospitals in Osogbo and Ogbomoso, southwestern Nigeria.

\section{METHODOLOGY}

Study Area and Population: The cross sectional study was conducted among consenting 333 (182 female and 151 male) patients with age range $0-52$ years attending Ladoke Akintola University of Technology Teaching Hospitals in Osogbo and Ogbomoso respectively. Osogbo and Ogbomoso are ancient cities of approximately 500,000 and 354,690 people respectively based on 2006 census provisional results, located in the heart of southwestern Nigeria.

Ethical Clearance: Concept of the study was duly explained to the subjects. Risks and benefits were also clearly stated and consent was duly signed. Ethical clearance and approval was obtained from Ethical Committee of LAUTECH Te a ching Hospital Os og b o (LTH/EC/2015/10/230). All consenting patients that attended the teaching hospital without age biased were selected.

Microscopy: Thick films were prepared according to the method of Chessbrough, (2002). Air-dried thick film was stained with $10 \%$ Giemsa solution for 15 minutes. Malaria parasites were examined using oil immersion lens objectives ( $\mathrm{x}$ 100) of a high quality microscope with an incandescent light source. Parasitaemia was expressed as the number of asexual forms of $\mathrm{P}$. falciparum per micro liter.
Methaemoglobin Reductase Test: Clean test tubes were arranged and labeled as test, normal, and deficient. Into the test tubes labeled test, 0.05 $\mathrm{ml}$ of sodium nitrite and $0.05 \mathrm{ml}$ of methylene blue reagents were dispensed. To the tubes labeled deficient, only $0.05 \mathrm{ml}$ sodium nitrite was added and in the test tube labeled normal, no reagent was dispensed. One (1.0) $\mathrm{ml}$ of the blood sample was then dispensed into all the tubes and mixed after which they were corked with cotton wool and incubated at $37{ }^{\circ} \mathrm{C}$ for $3 \mathrm{~h}$. At the end of the incubation, other three (3) clean test tubes were arranged and labeled as before (test, normal, deficient). Ten (10) $\mathrm{ml}$ of distilled water was dispensed into each of the test tubes. Then, $0.1 \mathrm{ml}$ of the respective incubated sample was transferred into each of the tubes accordingly. Colour change using spectrophotometer was observed and compared in the three test tubes (Cheesbrough, 2005).

\section{RESULTS}

Out of the 333 samples investigated, 283 (85\%) samples were found to be negative for malaria parasite, while $50(15 \%)$ were positive for malaria, which shows that the prevalence of malaria in these sites is $15 \%$. It was found that $34(10.2 \%)$ were found positive for malaria parasite among the female and $16(4.8 \%)$ among the male. This finding showed that there is significant difference between female and male in the malaria distribution at a p-value of 0.04 as shown in table 1.

Distribution of malaria parasite among different age group revealed that $6(1.8 \%)$ of children below one month were positive for malaria. It was observed that $8(2.4 \%)$ of participants within age 1-15 years were malarial parasite positive. Among participants within 16-20 years of age, $8(2.4 \%)$ were positive for malaria. Occurrence of 10 $(3.0 \%)$ and $4(1.2 \%)$ were observed in $21-36$ years and 37-52 years respectively. However, 14 (4.2\%) malarial parasite was found among the subjects within the age range $>52$ years. It was observed that there was a significant difference in the prevalence of malaria parasite with respect to age at p-value of (0.01) as shown in table 2.

The prevalence rate of G6PD deficiency using 
metheamoglobin reductase method was found to be $76(22.8 \%)$. Distribution of G6PD deficiency was observed to be $41(12.3 \%)$ and $35(10.5 \%)$ for male and female participants respectively. Overall, $257(77.2 \%)$ were found to have normal G6PD enzyme p-value (0.086), this showed that the relationship between G6PD deficiency and gender is not significant ( $\mathrm{p}$-value of 0.086 ) (Table 3).

The prevalence rate of G6PD deficiency compare with sex and age showed that 17 (5.1\%) were deficient in the $\leqslant 1$ year in which male has the highest frequency (11) compared to G6PD deficient female (6). Among the age range of 1-15 years, the prevalence rate was observed to be 6 $(1.8 \%)$ where G6PD deficient males were four (4) and females were two (2). Prevalence rate of 10 $(18.5 \%)$ was observed in the age range of $16-20$ years. Within the age group of $16-20$ years, males had the highest frequency (7) of G6PD deficiency compared to frequency of G6PD deficiency in females (3). It was observed in the age range 21-36 years, there was $24(31.6 \%)$ prevalence of G6PD deficiency with highest frequency of thirteen (13) in females while eleven (11) was obtained in male. In age group of 37-52 years, the prevalence rate of G6PD deficiency was $10(3.0 \%)$, with seven (7) as frequency in females and three (3) in males. The prevalence rate of G6PD deficiency drastically reduced in age range $\geqslant 52$ years with value of 9 $(2.7 \%)$ in which six $(6)$ was observed in males and three (3) in the female subjects as shown in figure 1.

This study showed that $215(64.8 \%)$ were found to have normal G6PD enzyme without malaria parasite. The overall prevalence of malaria observed in our study was $15 \%$ (Table 1 ). It was found that $67(20.2 \%)$ were G6PD deficient without malaria parasite. However, $8(2.4 \%)$ were found to be positive for malaria parasite and G6PD deficient. Statistically, there is no relationship between G6PD deficiency and malaria infection $(\mathrm{p}$-value $=0.227)$.

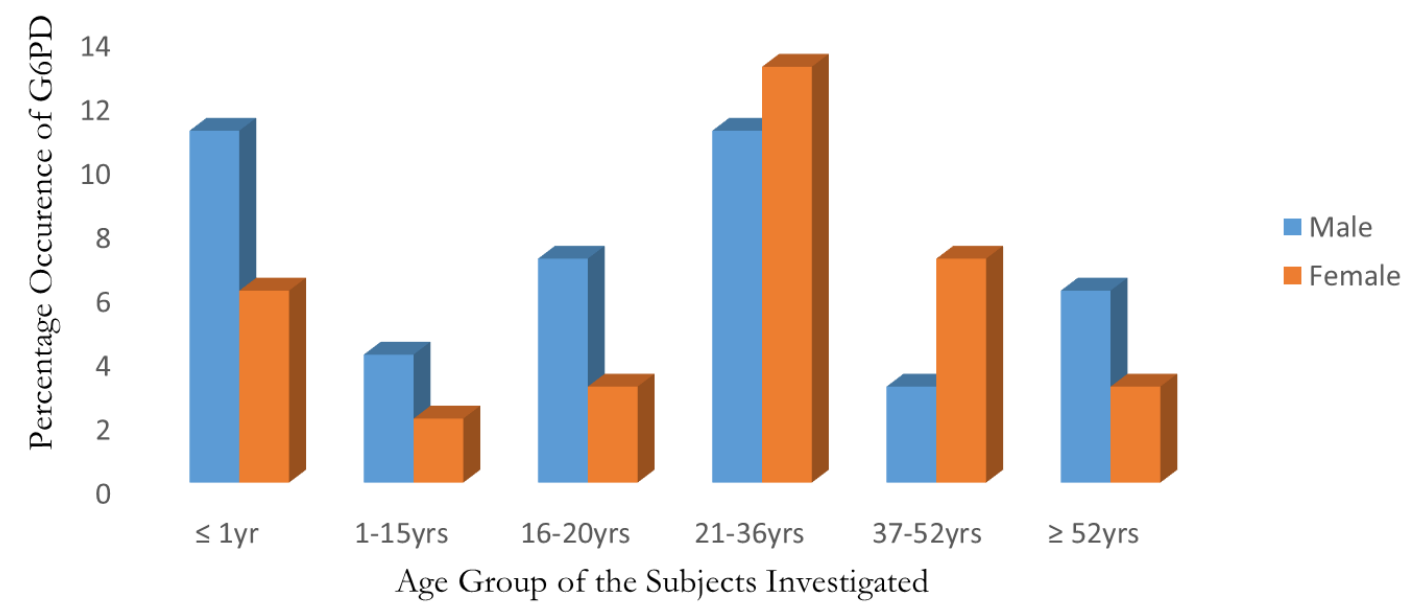

Figure 1: The prevalence of G6PD deficiency with respect to age and sex.

Table 1: Prevalence of Malaria Parasite based on Gender

\begin{tabular}{lllllll}
\hline & Negative & Positive & Total & df & $\mathrm{X}^{2}$ & P-value \\
\hline male & $148(44.4 \%)$ & $34(10.2 \%)$ & $182(54.7 \%)$ & 1 & 4.228 & 0.040 \\
Male & $135(40.5 \%)$ & $16(4.8 \%)$ & $151(45.3 \%)$ & & & \\
Total & $283(85.0 \%)$ & $50(15.0 \%)$ & $333(100 \%)$ & & &
\end{tabular}

p-value $=0.04$ 
Table 2: Prevalence of Malaria in Relationship with Age

\begin{tabular}{llllll}
\hline Age range & Negative & Positive & df & $\mathrm{X}^{2}$ & P-value \\
\hline$<$ 1year & $22(6.6 \%)$ & $6(1.8 \%)$ & & & \\
1-15year & $15(4.5 \%)$ & $8(2.4 \%)$ & & & \\
16-20year & $46(13.8 \%)$ & $8(2.4 \%)$ & 6 & 23.373 & 0.01 \\
21-36year & $108(32.4 \%)$ & $10(3.0 \%)$ & & & \\
37-52year & $58(17.4 \%)$ & $4(1.2 \%)$ & & & \\
$>52$ years & $34(10.2 \%)$ & $14(4.2 \%)$ & & & \\
Total & $283(85.0 \%)$ & $50(15.0 \%)$ & & & \\
\hline
\end{tabular}

Table 3: Prevalence of G6PD Deficiency with Gender

\begin{tabular}{lrcccc}
\hline & Normal & Deficient & df & $X^{2}$ & P-value \\
\hline Female & $147(44.1 \%)$ & $35(10.5 \%)$ & & & \\
Male & $110(33.0 \%)$ & $41(12.3 \%)$ & & & \\
Total & $257(77.2 \%)$ & $76(22.8 \%)$ & 1 & 2.940 & 0.086 \\
\hline
\end{tabular}

Table 4: Relationship between G6PD and Malaria

\begin{tabular}{lclccr}
\hline Malaria parasite & Normal & Deficient & df & $X^{2}$ & P-value \\
\hline Negative & $215(64.8 \%)$ & $67(20.2 \%)$ & & & \\
Positive & $42(84.9 \%)$ & $8(2.4 \%)$ & & & \\
Total & $257(77.4 \%)$ & $75(22.6 \%)$ & 1 & 1.462 & 0.227 \\
\hline
\end{tabular}

\section{DISCUSSION}

The prevalence of malaria as observed in this study was $15 \%$ of the total population investigated which is lower compared to the Nigeria Malaria Fact Sheet as reported by Nigeria Malaria Indicator Survey (NMIS, 2010) where $50 \%$ was reported as prevalence especially in urban areas. The reason for the reduction in prevalence level could be as a result of public awareness about malaria, distribution of mosquito net and environmental sanitation (involving clearing of bushes and cleaning of drainages) as recommended by NMIS (NMIS, 2010; Bello et al., 2016).

The prevalence rate of malaria among the female subjects $(10.2 \%)$ was higher compared to that of male $(4.8 \%)$. The difference in the prevalence is statistically significant ( $\mathrm{p}$-value of 0.04 ) between male and female. It was found out that prevalence of malaria was highest in the age range $>52$ years. This could be due to the fact that immune systems in individuals within this age range might be less efficient because of ageing. This study showed that there is significant association between infection rate of malaria and age $(p=0.01)$.

The prevalence of G6PD deficiency was $22.8 \%$ in this present study which is similar to $19.5 \%$ prevalence of G6PD deficiency reported by Akanni et al., (2010) in Osogbo among blood donors. In this study, prevalence of G6PD deficiency was $12.3 \%$ among male subjects while that of females was found to be $10.5 \%$. This finding is similar to $26 \%$ among males and $20 \%$ among females as earlier reported (Luzzatto and Notaro, 2001; Ademowo and Falusi, 2002; Egesie et al., 2008). The higher occurrence observed among the male subjects may be due to the fact that G6PD gene is X-linked and therefore G6PD deficiency is an X-linked disorder. In this study, it was observed that out of 27 neonatal samples ( $\leqslant$ 1year), 17 (5.1\%) individuals were found to be G6PD deficient. The prevalence of G6PD deficiency in this study was observed to be high among subjects within age range 16-20 years while the lowest prevalence occurred in age group of 1 15 years which is within the nursing and early 
adolescent age.

Nigeria is one of the subtropical countries where malaria is endemic. This deficiency may reduce malaria occurrence because of spontaneous decline and instability of G6PD enzymatic activities and also preventing malaria parasite from completing its life cycle (Howes et al., 2012). However, this study showed there is no association $(p$-value $=0.227)$ between G6PD and malaria parasites.

Correlating G6PD deficiency and the infection rate, it could be concluded that malaria parasite will be more active in G6PD deficient erythrocytes compare to G6PD normal erythrocytes. This was observed in our findings where it was found that $8(2.4 \%)$ of the subjects were found to be positive for malaria parasite and G6PD deficiency. The case fatality of the malaria infection may increase especially where there is cooccurrence of G6PD deficiency and malaria infection because of possibility of G6PD deficiency causing abnormal rupture of the red blood cell membrane with resultant hemolytic anemia.

\section{CONCLUSION}

The observation in this study gave the indication that there was significant prevalence of malaria among the female population. The occurrence of G6PD deficiency was high in male while the relationship between G6PD and malaria infection is not significant. This scenario can lead to high level of anaemic condition especially whenever it co-occurs with malaria. Therefore there is need for the health workers handling cases of malaria to take cognizance of some patients that may be anaemic which will not only complicate the infection but possibly lead to unexpected death.

\section{Acknowledgement}

Our sincere gratitude is expressed to the LAUTECH Teaching Hospitals in Osogbo and Ogbomoso for providing enabling environment and laboratory for the research work. Also, we thank all the participants for giving their consents towards this research work.

\section{Authors' Contribution}

NSA \& O design the experiment, OOS carry out the laboratory work, NSA, OJO and OOS interpreted the data. OJO \& NAS wrote the manuscript. All the authors approved the manuscript.

Declaration of Conflict of Interest: All authors declared that there is no conflict of interest.

\section{REFERENCES}

Ademowo, O.G. and Falusi, A.G. 2002. Molecular epidermiology and activities of erythrocytes G6PD variants in homogeneous Nigerian population. East African Journal, 79: 42-44.

Akanni, E.O., Oseni, B.S., Taiwo, V.O., Tijani, B.A., Tosan, E., Fakunle, E.E. and Mabayoje, V.O. 2010. Glucose 6 phosphate dehydrogenase deficiency in blood donors and jaundiced neonates in Osogbo, Nigeria. Journal of Medical Laboratory and Diagnosis, 1:1-4.

Bello I., Abdulsalami M. Sani, B. T., 2016. Prevalence of glucose-6-phosphate dehydrogenase deficiency among children aged 0-5 years infected with Plasmodium falciparum in Katsina State, Nigeria. Advances in Biochemistry, 4(6): 66-73. doi: 10.11648/j.ab.20160406.11

Beutler, E. 1983. Glucose-6-phosphate dehydrogenase deficiency. The Metabolic Basis of Inherited Disease, 5:1629-1653.

Cappellini, M.D. and Fiorelli, G. 2008. Glucose-6phosphate dehydrogenase deficiency. The Lancet, 371: 64-74.

Cheesbrough, M. 2002. Blood Transfusion Practice: Blood donation and Storage of blood. District Laboratory Practice in Tropical Countries, Pp: 352-353.

Cheesbrough, M. 2005. Blood Transfusion Practice: Blood donation and Storage of blood. District Laboratory Practice in Tropical Countries. Pp: 352-353.

Egesie, O.J., Joseph, D.E., Isiguzoro, I. and Egesie, U.G., 2008. Glucose 6phosphate dehydrogenase activities and deficiency in a population of Nigerian males. Nigerian Journal of Physiological Science, 23: 9-11.

GBD, 2013. Mortality and cause of death, collaborators 2014. Global, regional, and cause-specific mortality for 240 causes of 
death, 1990-2010: a systematic analysis for the Global burden of disease study. Lancet; 71 - 117 Vol. 385. Author: Antonio C. College of Public Health, University of Philippines Manila.

Haworth, J., Wernsdorfer W. and McGregor I., 1988. The global distribution of malaria and thepresent control effort. Malaria: Principles and Practice of Malariology. 2: 1379-1420.

Howes, R.E., Piel, F.B., Patil, A.P., Nyangiri, O.A., Gething, P.W., Dewi, M., Hogg, M.M., Battle, K.E., Padilla, C.D., Baird, J.K. and Hay, S.I. 2012. G6PD deficiency prevalence and estimates of affected populations in malaria endemic countries: a geostatistical model-based map. PLoS Med. 2012;9(11): https://doi.org/ 10.1371/journal.pmed.1001339.

Lukens, J.N. and Glader, B.E. 1999. Hereditary hemolytic anaemia sassociated with abnormalities of erythrocyte glycolysis and nucleotide metabolism. Wintrobe's Clinical Hematology, 10th edition. 11601175.

Luzzatto, L. 2010. Glucose-6-phosphate dehydrogenase (G6PD) deficiency: In the
Oxford Textbook of Medicine. Oxford University Press, USA. Pp: 5518/0 DOI:10.1093/med/9780199204854.003. 220512.

Luzzatto, L. and Notaro, R. 2001. Protecting against bad air. Science, 293: 442-443.

Nigeria Malaria Indicator Survey (NMIS), 2010. The DHS programme. Malaria fact sheets. Measure DHS Project. Pp: 43- 54.

Sutherland, C.J., Tanomsing, N. and Nolder, D. 2010. Two nonrecombining sympatric forms of the human malaria parasite Plasmodium ovale occur globally. Journal of Infectious Diseases. 201:1544-1550.

Viroj, W. 2005. Is the G6PD activity assay more cost effective than the methaemoglobin reduction test in screening for G6PD deficiency. Haematology. 8: 61-63.

Williams O, Gbadero D, Edowhorhu G, Brearley A, Slusher T, Lund TC (2013) Glucose-6Phosphate Dehydrogenase Deficiency in Nigerian Children. PLoS ONE 8(7): e68800. https://doi.org/10.1371 /journal.pone.0068800.

W.H.O. 2009. The world health report 2003: shaping the future. Geneva: Annex Table. 2: 156 . 